\title{
An instrument for online measurement of structural orientation in translucent polymer sheets
}

\author{
P K PALANISAMY*, D MANGAIYARKARASI and A RAMALINGAM \\ Centre for Laser Technology, Department of Physics, Anna University, Chennai 600 025, India
}

MS received 25 October 1999; revised 6 December 1999

\begin{abstract}
In many applications of polymer materials, a high tensile strength is required. There is a definite correlation between the degree of orientation of molecular chains achieved in the orientation process and the tensile strength obtained. In this work, we describe design and fabrication details of a device, 'Laser based polymer orientation grader', for online measurement of the degree of orientation to obtain a high consistent tensile strength as output. This instrument has been designed making skilful use of the optical anisotropic property of the oriented polymer strip. The principle is based on the fact that the spatial distribution of laser light passing through the translucent polymer sheet depends on the degree of orientation of long chain molecules in the sheet. The method has potential online application in monitoring and controlling of anisotropy in manufacturing processes.
\end{abstract}

Keywords. Online measurement; tensile strength; molecular orientation of long chains; anisotropy; scattering; diffraction.

\section{Introduction}

In many applications, polymer materials of a high tensile strength are required in thermo plastic industries. There is a definite correlation between the degree of orientation of molecular chains achieved in the process and the tensile strength obtained. The longitudinal orientation of chains is the most important factor for prevention of elongation and shrinkage of flat polymer strips, as strips with unoriented structures elongate more than the strips with longitudinally oriented structures. More than $90 \%$ of commercial nylon plastics are manufactured by condensation polymerization via epsilon-caprolactom dehydration (Yang and Lauke 1995) which typically has the disadvantage of a low degree of polymerization and wide molecular weight distribution. It has been reported that factors like orientation of molecules, per cent crystallinity, etc determine the constraints on the molecules. It was suggested that when the films are stretched under hot conditions, constraints on the molecules are greater in the direction where molecular orientation is maximum, resulting in lower expansion (Gohil 1993). Anisotropy arises from longitudinal orientation of long chains. The first publication on thermal anisotropy of polymer belts appeared in 1970. Recently an apparatus has been constructed for measuring simultaneously the thermal conductivity and viscosity of polymers at various shear rates and temperatures for controlling injection-moulding

\footnotetext{
*Author for correspondence
}

process to achieve the required anisotropy (Tavman 1997).

Already many techniques are available to measure the degree of anisotropy, e.g. wide-angle X-ray diffraction method is generally used to measure orientation in the crystalline regions. Measurement of tensile strength in two perpendicular directions in the polymer sheet is yet another method of measuring degree of structural anisotropy. These techniques cannot be used for online measurement of the degree of structural anisotropy. In this work, we describe design and fabrication details of a 'Laser based polymer orientation grader' device for online measurement of the degree of structural anisotropy enabling one to control the manufacturing process to obtain a high consistent tensile strength as output.

\section{Experimental}

\subsection{Light source}

Lasers are highly directional and coherent sources at precisely defined wavelengths. Laser-based measuring systems are highly reliable and accurate. Semiconductor diode laser, one form of laser system, has been used as the light source in this work. The diode laser generates stimulated emission of light caused by flow of electric current through a junction of $p$ and $n$ semiconductors. Wavelength is mainly in the near-IR region. The diode laser has the excellent feature of demonstrating stable performance for a long period of time. Compactness, 
simplicity and efficiency are the main advantages of semiconductor diode laser. It requires only $3 \mathrm{~V}$ d.c. power supply.

\subsection{Photodetectors}

The photodetector is a resistive transducer whose resistance changes as a function of external light. The best known and easiest-to-use type of light-sensitive device (photodetector) is the light-dependent resistor or LDR which uses the symbol shown in figure 1a. LDR operation relies on the fact that the resistance of cadmium sulphide (CdS) film varies with the intensity of the light falling on its face; the resistance is very high under dark conditions, and low under bright conditions. Figure $1 \mathrm{~b}$ shows the LDR's basic construction, which consists of a pair of metal film contacts separated by a snack-like track of cadmium sulphide film designed to give the maximum possible contact area with the two metal films. The structure is housed in a clear plastic case that gives free access to external light.

Practical LDRs are sensitive, inexpensive and readily available devices with good voltage and power handling capabilities, similar to those of a normal resistor. They are available in several sizes and package styles; the most popular size having a face diameter of roughly $10 \mathrm{~mm}$. Typically, such a device has a resistance of several megaohms under dark conditions, falling to about $900 \mathrm{R}$ at a light intensity of 100 Lux (typical of a well lit room) or about $30 \mathrm{R}$ at 8000 Lux (typical of bright sunlight).

\subsection{Measurement technique}

The measuring optical system consists of a semiconductor diode laser of $3 \mathrm{~mW}$ power, $670 \mathrm{~nm}$ wavelength as the optical source, and two pairs of light-dependent resistors belonging to the family of photodetectors described above. The principle of the instrument developed is based on the fact that the laser beam passing through the


Figure 1. a. LDR symbol and b. basic structure of LDR. translucent unoriented strip gives rise to diffused circular halo on the otherside of the strip due to diffusion as shown in figure $2 \mathrm{a}$; and if it passes through an oriented polymer strip, in addition to diffusion, diffraction also takes place. As a result in the direction perpendicular to the longitudinally oriented structure, elongated diffused light is received on the other side of the strip as shown in figures $2 b$ and $c$. This concept is made use of in the


Figure 2. Spatial distribution of laser light on passing through polymer sheet of a. zero\% molecular orientation, b. $40 \%$ molecular orientation and c. $70 \%$ molecular orientation. 
measurement of structural orientation in polymer sheets/ strips.

\section{Discussion}

\subsection{Structural orientation measurement}

From the semiconductor laser source, the coherent light beam is incident on the polymer strip under study. The light beam transmitted through the polymer strip is received by two pairs of detectors $D_{1}, D_{2}$ and $D_{3}, D_{4}$ positioned equidistant from the axis of the laser beam in perpendicular directions as shown in figure $3 \mathrm{a}$. The entire detector assembly is covered with red colour filter which allows only laser light to pass through and stops any stray light from reaching the detectors. The spatial distribution of transmitted light in horizontal and vertical directions are measured by the two pairs of detectors $D_{1}, D_{2}$ and $D_{3}$, $D_{4}$, respectively. The signal from these two pairs of detectors are fed to the differential amplifier after sufficient amplification. The circuit diagram of the device is shown in figure $3 \mathrm{~b}$.

When an unoriented polymer strip is placed in the path of the laser beam, both horizontal and vertical detectors receive the same amount of light energy and therefore the output from the differential amplifier is zero. Instead, when a sample of certain degree of orientation is intro-
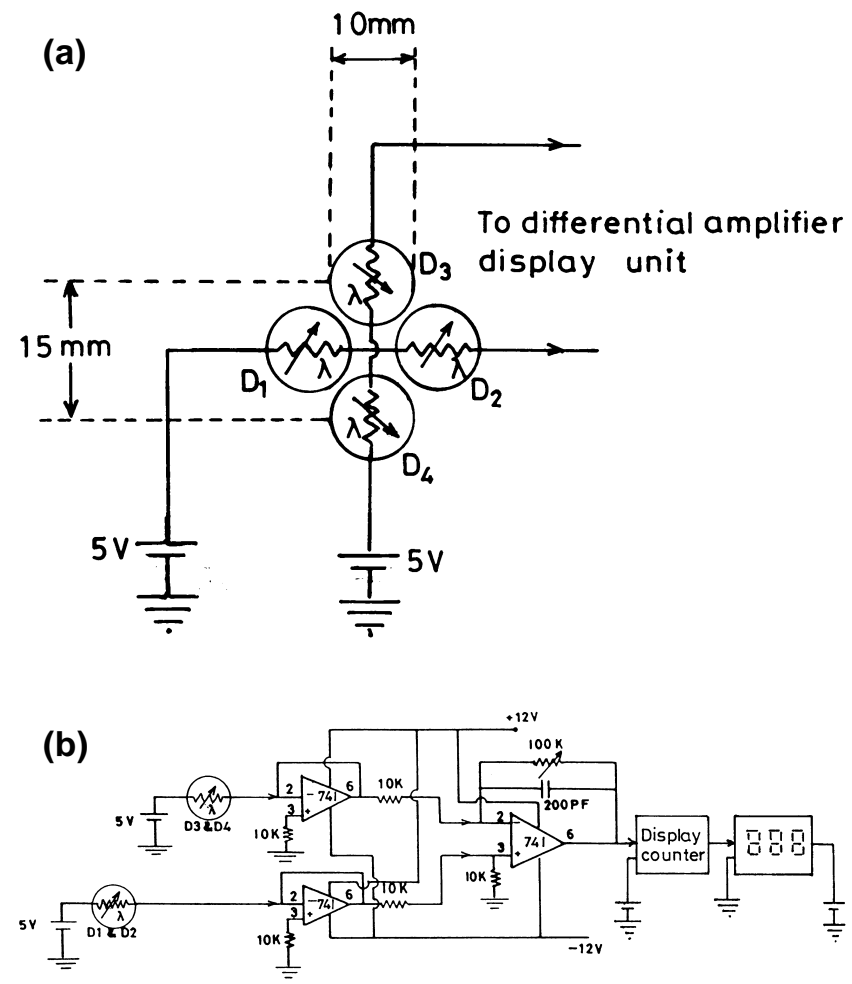

Figure 3. a. Layout of photodetectors, and b. circuit diagram of the detector-amplifier-display part for online measurement of structural orientation in polymer strips. duced, in addition to diffusion, diffraction also takes place resulting in elongated diffused light on the other side. The extent of elongation is proportional to the degree of orientation and the direction of elongation is perpendicular to the direction of orientation of the long chain molecules. Hence when the sample with longitudinal (vertical) molecular orientation is introduced, the horizontally positioned detectors $D_{1}, D_{2}$ receive more light than the other two detectors $D_{3}, D_{4}$. Because of the difference in the intensity of light received, the two pairs of detectors feed differing signals to the differential amplifier. The gain of the variable stage is adjusted to set for the known orientation value in the display with standard sample introduced. Once the instrument is calibrated, the signal response is found to be linear for all other samples of known values of degree of orientation. Now

Table 1. Measurement of structural orientation in the polymer strips (nylon 6).

\begin{tabular}{lcc}
\hline & \multicolumn{2}{c}{ Degree of structural orientation (\%) } \\
\cline { 2 - 3 } Nample & $\mathrm{a}$ & $\mathrm{b}$ \\
\hline 1. & $21-25$ & 22 \\
2. & $36-40$ & 39 \\
3. & $46-50$ & 47 \\
4. & $61-65$ & 64 \\
5. & $71-75$ & 71 \\
\hline
\end{tabular}

${ }^{\mathrm{a}}$ From tensile measurement technique; ${ }^{\mathbf{b}}$ from 'Laser based polymer orientation grader'.

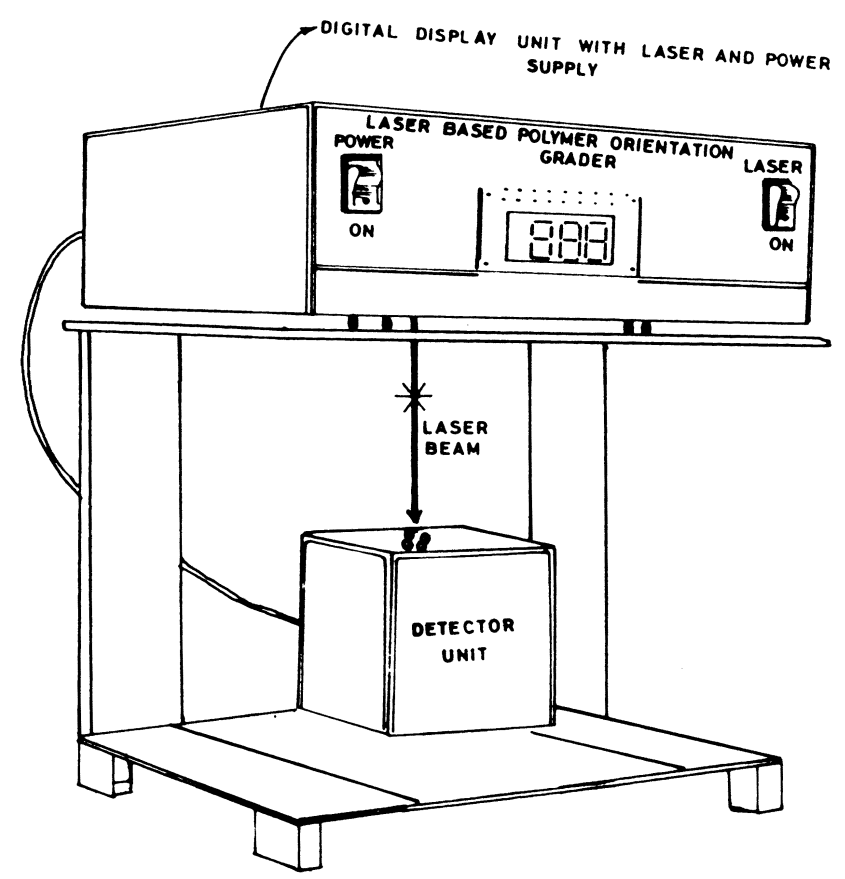

Figure 4. A sketch of the developed 'Laser based polymer orientation grader' device. 
the instrument is ready to measure the structural orientation in the unknown samples. On introducing the samples in the path of the laser in proper direction, the degree of structural orientation of long chain molecules in the polymer sheets is displayed directly in the display unit. It is important that the instrument is calibrated for the particular sample which is being monitored. The diffuse scattering, which gives the halo, depends on the porosity and the delustrant content of the material which could vary from sample to sample. In absence of such sample, though the absolute value of orientation measurement is not possible, relative orientation can be continuously measured.

In more than 50 nylon- 6 samples received from $\mathrm{K} \mathrm{F}$ Beltings private limited (Punjab), India, measurement of structural orientation of long chain molecules was done using this device and the results of these samples very well agreed with the data provided by the suppliers who have used tensile strength measurement technique to estimate the percentage structural orientation (table 1). A sketch of the device developed is shown in figure 4 . The entire device weighs only $1500 \mathrm{~g}$.

\section{Conclusion}

Though many techniques are available to measure the degree of structural orientation of long chain molecules in polymers based on different properties, this particular technique is well suited for online process. Once calibrated with standard sample, the instrument reads the absolute orientation in polymer strips. In absence of standard sample, relative orientation measurement helps in controlling the process. It has extremely high sensitivity, reliability and reproducibility. It provides a nondestructive technique for measuring structural orientation of long chain molecules, which is directly related to its tensile strength. This device measures the structural orientation in the polymer strip while on production; thus enabling one to control the manufacturing process.

\section{References}

Gohil R M 1993 J. Appl. Polym. Sci. 481649

Tavman I H 1997 Measurement Sci. \& Technol. 8287

Yang Z G and Lauke B 1995 J. Appl. Polym. Sci. 57679 